PROCEEDINGS OF THE

AMERICAN MATHEMATICAL SOCIETY

Volume 134, Number 11, November 2006, Pages 3353-3361

S 0002-9939(06)08410-3

Article electronically published on May 12, 2006

\title{
NONLINEAR CAUCHY PROBLEMS WITH SMALL ANALYTIC DATA
}

\author{
HIDESHI YAMANE
}

(Communicated by David S. Tartakoff)

\begin{abstract}
We study the lifespan of solutions to fully nonlinear Cauchy problems with small real- or complex-analytic data. Our proofs are based on the method of majorants and the fixed point theorem for a contraction mapping.
\end{abstract}

\section{INTRODUCTION}

The Cauchy problem for nonlinear wave equations with small data has been studied by many authors in the $\mathcal{C}^{\infty}$-category. They usually consider initial data with compact support and estimate the lifespan of a solution from below by using Fourier analysis. In particular, much attention has been paid to semilinear wave equations. Some monographs ([2, 4 4] and [5]) are available on this subject and detailed lists of references are found in them.

On the other hand, some results have been obtained about the Kirchhoff equation in the real-analytic category ([1] and [3]).

In the present paper, we consider fully nonlinear problems in the real- or complexanalytic category without hyperbolicity assumption. Our main tool is a combination of the fixed point technique and the method of majorants. We basically follow 6] and [3] with somewhat different notation.

Now we state our result.

Let $\Omega$ be an open set of $\mathbb{R}_{x}^{n}, x=\left(x_{1}, \ldots, x_{n}\right)$. A $\mathcal{C}^{\infty}$-function $\varphi(x)$ on $\Omega$ is said to be uniformly analytic on $\Omega$ if it has the uniform bound below:

$$
\exists C>0, \forall \alpha \in \mathbb{N}^{n}, \sup _{x \in \Omega}\left|\partial^{\alpha} \varphi(x)\right| \leq C^{|\alpha|+1}|\alpha| ! .
$$

Note that the right-hand side is equivalent to the Cauchy-type bound $C^{|\alpha|+1} \alpha$ ! up to the choice of $C$.

We define the function space $A(\Omega)$ to be the totality of uniformly analytic functions on $\Omega$. It is trivial that $A(\Omega)$ is closed under differentiation.

Let $t$ be a point of $\mathbb{R}$. For $T>0$, the open interval ] $-T, T$ [ is denoted by $I_{T}$. We set $\Omega_{T}=I_{T} \times \Omega$.

For $k \in \mathbb{N}$, a continuous function $u(t, x)$ on $\Omega_{T}=I_{T} \times \Omega$ is said to belong to $\mathcal{C}^{k}(T ; A(\Omega))$ if

(i) $\forall j \in\{0, \ldots, k\}, \forall \alpha \in \mathbb{N}^{n}, \partial_{t}^{j} \partial^{\alpha} u \in \mathcal{C}\left(\Omega_{T}\right)$,

Received by the editors November 23, 2004 and, in revised form, June 6, 2005.

2000 Mathematics Subject Classification. Primary 35A05, 35L70.

Key words and phrases. Nonlinear wave equations, analytic functions.

(C)2006 American Mathematical Society Reverts to public domain 28 years from publication 
(ii) $\left.\forall T^{\prime} \in\right] 0, T\left[, \exists C=C_{T^{\prime}}>0, \forall j \in\{0, \ldots, k\}, \forall \alpha \in \mathbb{N}^{n}\right.$,

$$
\sup _{|t| \leq T^{\prime}, x \in \Omega}\left|\partial_{t}^{j} \partial^{\alpha} u(t, x)\right| \leq C^{|\alpha|+1}|\alpha| !
$$

Let $P=P(\partial)=\sum_{k=1}^{n} \sum_{j=1}^{k} p_{j k} \partial_{j} \partial_{k}$ be a second-order linear partial differential operator with constant coefficients, where $\partial_{j}=\partial / \partial x_{j}$ and $p_{j k} \in \mathbb{C}$. We consider the following Cauchy problem for a fully nonlinear equation:

$$
\left\{\begin{array}{l}
\left(\partial_{t}^{2}-P(\partial)\right) u(t, x)=f\left(\nabla u(t, x), \nabla^{2} u(t, x)\right) \\
u(0, x)=\varphi(x), \partial_{t} u(0, x)=\psi(x)
\end{array}\right.
$$

where $\nabla u(t, x)=\left(\partial_{j} u\right)_{1 \leq j \leq n}$ and $\nabla^{2} u(t, x)=\left(\partial_{j} \partial_{k} u\right)_{1 \leq j \leq k \leq n}$. Here $\varphi(x)$ and $\psi(x)$ are uniformly analytic in an open subset $\Omega$ of $\mathbb{R}_{x}^{n}$. We assume that $f(X)$ is real-analytic near $X=0 \in \mathbb{R}^{N}, N=\left(n^{2}+3 n\right) / 2$, and vanishes of second order at $X=0$. The right-hand side in $(\mathrm{CP})$ does not depend on the variables $t, x$, or on the unknown function $u$ and its time derivative $\partial_{t} u$. This condition makes it small in the sense described below (See Proposition 2.5 and the proof of Theorem 1.1 in \$4)

We shall study the lifespan of a solution when the data are small in some sense.

Theorem 1.1. There exist $\mu>0$ and $\varepsilon_{0}>0$ such that the following holds for all $\varepsilon$ with $0<\varepsilon \leq \varepsilon_{0}$ :

$$
\text { If } \sup _{x \in \Omega}\left|\partial^{\alpha} \bar{\varphi}\right| \leq \varepsilon^{|\alpha|+1}|\alpha| \text { ! and } \sup _{x \in \Omega}\left|\partial^{\alpha} \psi\right| \leq \varepsilon^{|\alpha|+1}|\alpha| \text { ! for all } \alpha \in \mathbb{N}^{n} \text {, then (CP) }
$$
has a solution $u(t, x) \in \mathcal{C}^{2}(T ; A(\Omega))$ for $T=\mu / \varepsilon$.

Roughly speaking, the bound on $\varphi$ and $\psi$ is equivalent to saying that they can be analytically continued to a large open set in $\mathbb{C}^{n}$ and have small modulus there.

Note that [7] shows that a hyperbolicity condition is necessary for a nonlinear Cauchy problem to be well posed.

Next, we shall state a complex-analytic version, in which $t$ and $x$ are both complex variables.

Let $U$ be an open set of $\mathbb{C}_{x}^{n}\left(\right.$ not $\left.\mathbb{R}^{n}\right)$. We consider $(\mathrm{CP})$ again, but now $\varphi(x)$ and $\psi(x)$ are both assumed to be complex-analytic functions on $U$. Naturally we try to find a solution which is complex-analytic in $(t, x)$.

For $T>0$, set $B_{T}=\{t \in \mathbb{C} ;|t|<T\}$.

Theorem 1.2. There exist $\mu>0$ and $\varepsilon_{0}>0$ such that the following holds for all $\varepsilon$ with $0<\varepsilon \leq \varepsilon_{0}$ :

$$
\text { If } \sup _{x \in U}\left|\partial^{\alpha} \varphi\right| \leq \varepsilon^{|\alpha|+1}|\alpha| \text { ! and } \sup _{x \in U}\left|\partial^{\alpha} \psi\right| \leq \varepsilon^{|\alpha|+1}|\alpha| \text { ! for all } \alpha \in \mathbb{N}^{n} \text {, then (CP) }
$$

has a unique solution $u(t, x)$ which is complex-analytic on $B_{T} \times U$ for $T=\mu / \varepsilon$ and satisfies the following estimate: for all $T^{\prime}$ with $0<T^{\prime}<T=\mu / \varepsilon$, there exists $C=C_{T^{\prime}}>0$ such that

$$
\sup _{|t| \leq T^{\prime}, x \in U}\left|\partial^{\alpha} u(t, x)\right| \leq C^{|\alpha|+1}|\alpha| !
$$

for $\alpha \in \mathbb{N}^{n}$. (An estimate on $\partial_{t}^{j} \partial^{\alpha} u(t, x)$ can be obtained by using Cauchy's inequality.) 


\section{The Banach algebra $\mathcal{G}_{T, \zeta}(\Omega)$}

Some material in this and the next sections has already appeared in [6] or [3, possibly in a different formulation. We present proofs here for the reader's convenience.

Let $f(X)=\sum_{k=0}^{\infty} a_{k} X^{k}$ and $g(X)=\sum_{k=0}^{\infty} b_{k} X^{k}$ be two formal series with $a_{k} \in \mathbb{R}, b_{k} \in \mathbb{R}_{+}$. Here $\mathbb{R}_{+}$is the totality of nonnegative real numbers. We write $f(X) \ll g(X)$ if $\left|a_{k}\right| \leq b_{k}$ for all $k \geq 0$.

If $0 \ll f(X) \ll g(X)$ and $p \geq 0$ is smaller than the radius of convergence of $g$, then we have $0 \ll f(X+p) \ll g(X+p)$. In fact the assumption $0 \leq\left|a_{k}\right| \leq b_{k}$ implies $0 \leq\left|\sum a_{k} p^{k}\right| \leq \sum b_{k} p^{k}, 0 \leq\left|\sum k a_{k} p^{k-1}\right| \leq \sum k b_{k} p^{k-1}, 0 \leq\left|\sum k(k-1) a_{k} p^{k-2}\right| \leq$ $\sum k(k-1) b_{k} p^{k-2}, \ldots$

For a formal power series $f(X)=\sum_{k=0}^{\infty} a_{k} X^{k}$, set

$$
\begin{aligned}
& D f(X)=\sum_{k=1}^{\infty} k a_{k} X^{k-1}=\sum_{k=0}^{\infty}(k+1) a_{k+1} X^{k}, \\
& D^{-1} f(X)=\sum_{k=0}^{\infty} \frac{a_{k}}{k+1} X^{k+1}=\sum_{k=1}^{\infty} \frac{a_{k-1}}{k} X^{k} .
\end{aligned}
$$

We have $D D^{-1} f(X)=f(X)$, but $D^{-1} D f(X)=\sum_{k=1}^{\infty} a_{k} X^{k} \neq f(X)$.

Set $\varphi(X)=\frac{1}{K} \sum_{k=0}^{\infty} \frac{X^{k}}{(k+1)^{2}}, K=4 \pi^{2} / 3$. It is a series due to Lax. It can be proved that $\varphi^{2}(X) \ll \varphi(X)$. Hence we have $0 \ll \varphi^{2}(X+p) \ll \varphi(X+p)$ if $p \geq 0$.

Assume that $k \leq 0, a \geq 0, b \geq 0, k+a \leq 0$. Then $D^{k} D^{a+b} \varphi(X)$ is obtained by cutting off the terms of degree $<-k$ from $D^{b} D^{k+a} \varphi(X)$. We have

$$
D^{k} D^{a+b} \varphi(X) \ll D^{b} D^{k+a} \varphi(X) .
$$

Since $k+a \leq 0$, Lemma 2.5 of [6] implies that $D^{k+a} \varphi(X) \ll c^{k+a} \varphi(X)$ with $c=2 / 9$ (our $c$ is the reciprocal of Wagschal's). Hence

$$
D^{b} D^{k+a} \varphi(X) \ll c^{k+a} D^{b} \varphi(X) .
$$

A combination of (11) and (2) shows that

$$
D^{k} D^{a+b} \varphi(X) \ll c^{k+a} D^{b} \varphi(X) .
$$

Passing from an indeterminate $X$ to a definite value $X_{0}$, we get

Proposition 2.1. Assume $a \geq 0, b \geq 0, k+a \leq 0$ and $0 \leq X_{0}<1$; we have

$$
D^{k} D^{a+b} \varphi\left(X_{0}\right) \leq c^{k+a} D^{b} \varphi\left(X_{0}\right) .
$$

If $\zeta>0$, then a continuous function $u(t, x)$ on $\Omega_{T}$ is said to be an element of $\mathcal{G}_{T, \zeta}(\Omega)$ if it is infinitely differentiable in $x$ and there exists a constant $C>0$ such that

$$
\forall \alpha \in \mathbb{N}^{n}, \forall t \in I_{T}, \quad \sup _{x \in \Omega}\left|\partial^{\alpha} u(t, x)\right| \leq C \zeta^{|\alpha|} D^{|\alpha|} \varphi(|t| / T),
$$

where $\partial^{\alpha}=\partial^{\alpha_{1}+\cdots+\alpha_{n}} / \partial x_{1}^{\alpha_{1}} \cdots \partial x_{n}^{\alpha_{n}}$.

We set

$$
\varphi_{T, \zeta}(t, x)=\varphi\left(\frac{|t|}{T}+\zeta \sum_{j=1}^{n} x_{j}\right) .
$$


Then we have $\partial^{\alpha} \varphi_{T, \zeta}(t, 0)=\zeta^{|\alpha|} D^{|\alpha|} \varphi(|t| / T)$, which is a factor of the right-hand side of (4). We denote (4) by

$$
u(t, x) \lll C \varphi_{T, \zeta}(t, x) .
$$

We define the norm $\|u\|$ to be the infimum of such $C$ 's.

Proposition 2.2. $\mathcal{G}_{T, \zeta}(\Omega)$ becomes a Banach space.

Proof. Let $\mathcal{C}^{0, \infty}\left(\Omega_{T}\right)$ be the space of continuous functions on $\Omega_{T}$ which are infinitely differentiable in $x$. For a compact subset $K$ of $\Omega_{T}$ and $\alpha \in \mathbb{N}^{n}$, set $p_{\alpha, K}(u(t, x))=$ $\sup _{K}\left|\partial^{\alpha} u(t, x)\right|$. Then $\mathcal{C}^{0, \infty}\left(\Omega_{T}\right)$ becomes a Fréchet space with these norms.

Obviously we have $\mathcal{G}_{T, \zeta}(\Omega) \subset \mathcal{C}^{0, \infty}\left(\Omega_{T}\right)$, and the canonical injection is continuous, because

$$
\sup _{|t| \leq T^{\prime}, x \in \Omega}\left|\partial^{\alpha} u(t, x)\right| \leq\|u\| \zeta^{|\alpha|} D^{|\alpha|} \varphi\left(T^{\prime} / T\right), 0<T^{\prime}<T .
$$

If $\left(u_{k}\right)$ is a Cauchy sequence in $\mathcal{G}_{T, \zeta}(\Omega)$, it converges to a limit $u$ in the Fréchet space $\mathcal{C}^{0, \infty}\left(\Omega_{T}\right)$. We have only to prove that $u \in \mathcal{G}_{T, \zeta}(\Omega)$ and that $\left(u_{k}\right)$ converges to $u$ in $\mathcal{G}_{T, \zeta}(\Omega)$.

For all $\varepsilon>0$, there exists $k \in \mathbb{N}$ such that $\left\|u_{p}-u_{q}\right\| \leq \varepsilon$ if $p, q \geq k$. In other words, for all $\alpha \in \mathbb{N}^{n}$ we have

$$
\sup _{x \in \Omega}\left|\partial^{\alpha} u_{p}-\partial^{\alpha} u_{q}\right| \leq \varepsilon \zeta^{|\alpha|} D^{|\alpha|} \varphi(|t| / T) .
$$

Since $\partial^{\alpha} u_{p}(t, x) \rightarrow \partial^{\alpha} u(t, x)$ for all $(t, x) \in \Omega_{T}$, we get

$$
\sup _{x \in \Omega}\left|\partial^{\alpha} u_{p}-\partial^{\alpha} u\right| \leq \varepsilon \zeta^{|\alpha|} D^{|\alpha|} \varphi(|t| / T) .
$$

This means that $u \in \mathcal{G}_{T, \zeta}(\Omega)$ and that $\left(u_{k}\right)$ converges to $u$ in $\mathcal{G}_{T, \zeta}(\Omega)$.

Note that our $\varphi_{T, \zeta}(t, x)$ and $\mathcal{G}_{T, \zeta}(\Omega)$ are different from $\Phi_{T, \zeta}(t, x)$ and $G_{T, \zeta}\left(\Omega_{T}\right)$ of [3]. It affects the formulation of Proposition 2.5. In the present paper we employ the unfamiliar symbol $\ll$ in dealing with estimates global in $x$, in contrast to $\ll$ which only gives local information.

Proposition 2.3. $\mathcal{G}_{T, \zeta}(\Omega)$ is a Banach algebra.

Proof. Since $0 \ll \varphi^{2}(X+p) \ll \varphi(X+p)$ for $p \in[0,1]$, we have for $t \in I_{T}$,

$$
\partial^{\alpha}\left(\varphi_{T, \zeta}^{2}\right)(t, 0) \leq \partial^{\alpha} \varphi_{T, \zeta}(t, 0) .
$$

If $u(t, x) \lll C_{1} \varphi_{T, \zeta}(t, x), v(t, x) \lll C_{2} \varphi_{T, \zeta}(t, x)$, then

$$
\left\{\begin{array}{l}
\sup _{x \in \Omega}\left|\partial^{\alpha} u(t, x)\right| \leq C_{1} \partial^{\alpha} \varphi_{T, \zeta}(t, 0), \\
\sup _{x \in \Omega}\left|\partial^{\alpha} v(t, x)\right| \leq C_{2} \partial^{\alpha} \varphi_{T, \zeta}(t, 0) .
\end{array}\right.
$$

Therefore

$$
\begin{aligned}
& \sup _{x \in \Omega}\left|\partial^{\alpha}(u v)(t, x)\right| \leq \sum_{\beta \leq \alpha} \sup _{x \in \Omega}\left|\left(\begin{array}{l}
\alpha \\
\beta
\end{array}\right)\left(\partial^{\alpha-\beta} u \cdot \partial^{\beta} v\right)(t, x)\right| \\
& \leq C_{1} C_{2} \sum_{\beta \leq \alpha}\left(\begin{array}{l}
\alpha \\
\beta
\end{array}\right) \partial^{\alpha-\beta} \varphi_{T, \zeta}(t, 0) \cdot \partial^{\beta} \varphi_{T, \zeta}(t, 0)=C_{1} C_{2} \partial^{\alpha}\left(\varphi_{T, \zeta}^{2}\right)(t, 0) \\
& \leq C_{1} C_{2} \partial^{\alpha} \varphi_{T, \zeta}(t, 0) .
\end{aligned}
$$

Hence $u v \lll C_{1} C_{2} \varphi_{T, \zeta}$. This implies that $\|u v\| \leq\|u\|\|v\|$. 
We equip the direct sum $\bigoplus^{N} \mathcal{G}_{T, \zeta}(\Omega)$ with the norm $\|\cdot\|_{N}$ defined by

$$
\begin{aligned}
& \|\vec{\tau}(t, x)\|_{N}=\max _{j=1, \ldots, N}\left\|\tau_{j}(t, x)\right\|, \\
& \vec{\tau}(t, x)=\left(\tau_{1}(t, x), \ldots, \tau_{N}(t, x)\right) \in \bigoplus^{N} \mathcal{G}_{T, \zeta}(\Omega) .
\end{aligned}
$$

Proposition 2.4. Let $f(X)=f\left(X_{1}, \ldots, X_{N}\right)=\sum_{|\alpha| \geq 2}^{\infty} a_{\alpha} X^{\alpha}$ be a convergent power series which vanishes of second order at $X=0$. If $\vec{\tau}(t, x), \vec{\sigma}(t, x) \in \bigoplus^{N} \mathcal{G}_{T, \zeta}(\Omega)$ have sufficiently small norms, then $f(\vec{\tau}(t, x))$ and $f(\vec{\sigma}(t, x))$ are well defined as elements of $\mathcal{G}_{T, \zeta}(\Omega)$. Moreover, there exist positive constants $C_{f}$ and $C_{f}^{\prime}$ depending only on $f$ and independent of $\vec{\tau}, \vec{\sigma}, T, \zeta$ and $\Omega$ such that

$$
\begin{aligned}
& \|f(\vec{\tau}(t, x))\| \leq C_{f}\|\vec{\tau}\|_{N}^{2}, \\
& \|f(\vec{\tau}(t, x))-f(\vec{\sigma}(t, x))\| \leq C_{f}^{\prime}\|\vec{\tau}-\vec{\sigma}\|_{N}\left(\|\vec{\tau}\|_{N}+\|\vec{\sigma}\|_{N}\right) .
\end{aligned}
$$

Proof. By Proposition 2.3, we have

$$
f(\vec{\tau})=\sum_{|\alpha| \geq 2}^{\infty} a_{\alpha} \vec{\tau}^{\alpha} \lll \sum_{|\alpha| \geq 2}^{\infty}\left|a_{\alpha}\right|\left\|\tau_{1}\right\|^{\alpha_{1}} \cdots\left\|\tau_{n}\right\|^{\alpha_{N}} \varphi_{T, \zeta} .
$$

We find that $\|f(\vec{\tau}(t, x))\| \leq C_{f}\|\vec{\tau}\|_{N}^{2}$ for some $C_{f}$ if $\|\vec{\tau}\|_{N}$ is sufficiently small.

We have $f(Y)-f(X)=(Y-X) \cdot g(X, Y)$ for a vector-valued real-analytic function $g(X, Y)=\int_{0}^{1} \nabla f((1-t) X+t Y) d t$. Since $g(0,0)=0$, the inequality $\|f(\vec{\tau}(t, x))-f(\vec{\sigma}(t, x))\| \leq C_{f}^{\prime}\|\vec{\tau}-\vec{\sigma}\|_{N}\left(\|\vec{\tau}\|_{N}+\|\vec{\sigma}\|_{N}\right)$ follows.

Set $\partial_{t}^{-1} u(t, x)=\int_{0}^{t} u(s, x) d s$.

Proposition 2.5. For all $(k, \alpha) \in(-\mathbb{N}) \times \mathbb{N}^{n}$ with $k+|\alpha| \leq 0$, there exists a constant $C_{k,|\alpha|}>0$ such that $\partial_{t}^{k} \partial^{\alpha}$ is an endomorphism of the Banach space $\mathcal{G}_{T, \zeta}(\Omega)$ and its norm is not larger than $C_{k,|\alpha|} T^{-k} \zeta^{|\alpha|}$.

Proof. We fix $\alpha$. If $u \in \mathcal{G}_{T, \zeta}(\Omega)$, we have for all $\beta \in \mathbb{N}^{n}$,

$$
\sup _{x \in \Omega}\left|\partial^{\alpha+\beta} u(t, x)\right| \leq\|u\| \zeta^{|\alpha+\beta|} D^{|\alpha+\beta|} \varphi(|t| / T) .
$$

Then by Proposition 2.1 we obtain the following estimate, in which we choose $\pm \partial_{t}$ if $\pm t \geq 0$ :

$$
\begin{aligned}
\sup _{x \in \Omega}\left|\partial_{t}^{k} \partial^{\alpha+\beta} u(t, x)\right| & \leq\|u\| \zeta^{|\alpha+\beta|}\left( \pm \partial_{t}\right)^{k}\left\{D^{|\alpha+\beta|} \varphi(|t| / T)\right\} \\
& \leq\|u\| T^{-k} \zeta^{|\alpha+\beta|} D^{k} D^{|\alpha+\beta|} \varphi(|t| / T) \\
& \leq\|u\| c^{k+|\alpha|} T^{-k} \zeta^{|\alpha|} \cdot \zeta^{|\beta|} D^{|\beta|} \varphi(|t| / T) .
\end{aligned}
$$

We have shown that

$$
\partial_{t}^{k} \partial^{\alpha} u(t, x) \lll\|u\| c^{k+|\alpha|} T^{-k} \zeta^{|\alpha|} \varphi_{T, \zeta}(t, x),
$$

because $\partial_{t}$ and $\partial^{\beta}$ commute. 


\section{UNIFORMLY ANALYTIC FUNCTIONS}

The spaces $A(\Omega)$ and $\mathcal{C}^{k}(T ; A(\Omega))$ have been defined in the first section. Recall condition (ii) in the definition of the latter, which is only locally uniform in $t$. This condition has been chosen so that the following proposition may hold. Note that $D^{k} \varphi(1)$ diverges if $k \geq 1$.

Proposition 3.1. $\forall T>0, \forall \zeta>0, \mathcal{G}_{T, \zeta}(\Omega) \subset \mathcal{C}(T ; A(\Omega))$.

To formulate an almost converse inclusion, we introduce the following notation. If $\varphi(x) \in A(\Omega)$, there exist positive constants $p(\varphi)>0$ and $q(\varphi)>0$ such that

$$
\forall \alpha \in \mathbb{N}^{n}, \sup _{x \in \Omega}\left|\partial^{\alpha} \varphi(x)\right| \leq p(\varphi) q(\varphi)^{|\alpha|}|\alpha| !
$$

They are not unique.

Remark 3.2. If $\Omega$ is star-shaped and $\varphi(x) \in A(\Omega)$, we set $\varphi_{\varepsilon}(x)=\varepsilon \varphi(\varepsilon x)$. Then we can take $p\left(\varphi_{\varepsilon}\right)=\varepsilon p(\varphi), q\left(\varphi_{\varepsilon}\right)=\varepsilon q(\varphi)$.

Proposition 3.3. Assume that $\varphi(x) \in A(\Omega)$ satisfies $\sup _{x \in \Omega}\left|\partial^{\alpha} \varphi\right| \leq \varepsilon^{|\alpha|+1}|\alpha|$ !. (We can take $p(\varphi)=q(\varphi)=\varepsilon$.) Then we can take

$$
\begin{aligned}
& p\left(\partial_{j} \varphi\right)=\varepsilon^{2}, p\left(\partial_{j} \partial_{k} \varphi\right)=3 \varepsilon^{3}, p\left(\partial_{j} \partial_{k} \partial_{\ell} \varphi\right)=15 \varepsilon^{4}, \\
& q\left(\partial_{j} \varphi\right)=q\left(\partial_{j} \partial_{k} \varphi\right)=q\left(\partial_{j} \partial_{k} \partial_{\ell} \varphi\right)=2 \varepsilon
\end{aligned}
$$

Proof. We have

$$
\sup _{x \in \Omega}\left|\partial^{\alpha}\left(\partial_{j} \varphi\right)\right| \leq \varepsilon^{|\alpha|+2}(|\alpha|+1) !=\frac{|\alpha|+1}{2^{|\alpha|}} \varepsilon^{2} \cdot(2 \varepsilon)^{|\alpha|}|\alpha| ! .
$$

Then we employ the fact that $j / 2^{j-1} \leq 1$ for $j \geq 1$.

Next we have

$$
\sup _{x \in \Omega}\left|\partial^{\alpha}\left(\partial_{j} \partial_{k} \varphi\right)\right| \leq \varepsilon^{|\alpha|+3}(|\alpha|+2) ! \leq \frac{(|\alpha|+2)(|\alpha|+1)}{2^{|\alpha|}} \varepsilon^{3} \cdot(2 \varepsilon)^{|\alpha|}|\alpha| ! .
$$

Then we employ the fact that $j(j+1) / 2^{j-1} \leq 3$ for $j \geq 1$.

The assertion about $\partial_{j} \partial_{k} \partial_{\ell} \varphi$ can be proved in a similar way.

Proposition 3.4. If $\psi(x) \in A(\Omega)$, then for all $T>0$ and for all $\zeta \geq e^{2} q(\psi)$, we have $\psi \in \mathcal{G}_{T, \zeta}(\Omega)$ and $\|\psi\| \leq K p(\psi)$.

Proof. For all $\alpha \in \mathbb{N}^{n}$, we have

$$
(|\alpha|+1)^{2} D^{|\alpha|} \varphi(|t| / T) \geq(|\alpha|+1)^{2} D^{|\alpha|} \varphi(0)=K^{-1}|\alpha| !
$$

and $(|\alpha|+1)^{2} \leq e^{2|\alpha|}$. Hence we obtain

$$
|\alpha| ! \leq K e^{2|\alpha|} D^{|\alpha|} \varphi(|t| / T) .
$$

On the other hand, $\psi(x) \in A(\Omega)$ satisfies

$$
\sup _{x \in \Omega}\left|\partial^{\alpha} \psi(x)\right| \leq p(\psi) q(\psi)^{|\alpha|}|\alpha| !
$$

By (5) and (6), we find that

$$
\sup _{x \in \Omega}\left|\partial^{\alpha} \psi(x)\right| \leq\{K p(\psi)\} \cdot\left\{e^{2} q(\psi)\right\}^{|\alpha|} D^{|\alpha|} \varphi(|t| / T) .
$$

This completes the proof. 


\section{Proofs of the TheOREMS}

Proof of Theorem 1.1. Set $v(t, x)=u(t, x)-\varphi(x)-t \psi(x)$. Then $v(0, x)=\partial_{t} v(0, x)$ $=0$ and

$$
\partial_{t}^{2} v=P(v+\varphi+t \psi)+f\left(\nabla^{1,2}(v+\varphi+t \psi)\right)
$$

where we set $\nabla^{1,2} u=\left(\nabla u, \nabla^{2} u\right)$ for simplicity.

Next we set $w=\partial_{t}^{2} v$. Then $v=\partial_{t}^{-2} w$ and $(\mathrm{CP})$ is reduced to $w=\mathcal{L}(w)$, where we define the mapping $\mathcal{L}$ by

$$
\mathcal{L}(w)=P\left(\partial_{t}^{-2} w+\varphi+t \psi\right)+f\left(\nabla^{1,2}\left(\partial_{t}^{-2} w+\varphi+t \psi\right)\right) .
$$

We shall find a fixed point $w$ of $\mathcal{L}$ in a suitable complete metric space by showing that $\mathcal{L}$ is a contraction.

We assume that $w \in \mathcal{G}_{T, \zeta}(\Omega)$, where $T$ and $\zeta$ are to be specified later.

By Propositions 2.5. we have

$$
\left\|P \partial_{t}^{-2} w\right\| \leq A\|w\|, \quad A:=C_{P} C_{-2,2} T^{2} \zeta^{2},
$$

where $C_{P}=\sum\left|p_{j k}\right|$. By Propositions 3.3 and 3.4, if $\zeta \geq 2 e^{2} \varepsilon$, we have

$$
\|P(\varphi+t \psi)\| \leq B, \quad B:=3 C_{P} K(1+T) \varepsilon^{3} .
$$

The nonlinear term is estimated by using Propositions 2.4, 3.3 and 3.4 If $\zeta \geq$ $2 e^{2} \varepsilon$ we have

$$
\begin{aligned}
& \left\|f\left(\nabla^{1,2}\left(\partial_{t}^{-2} w+\varphi+t \psi\right)\right)\right\| \\
& \leq C_{f}\left(\left\|\nabla^{1,2} \partial_{t}^{-2} w\right\|_{N}+\left\|\nabla^{1,2}(\varphi+t \psi)\right\|_{N}\right)^{2} \\
& \leq C_{f}\left\{\max \left(C_{-2,1} T^{2} \zeta, C_{-2,2} T^{2} \zeta^{2}\right)\|w\|+K(1+T) \varepsilon^{2}\right\}^{2} \\
& =\left(A^{\prime}\|w\|+B^{\prime}\right)^{2},
\end{aligned}
$$

where $A^{\prime}:=\sqrt{C_{f}} \max \left(C_{-2,1} T^{2} \zeta, C_{-2,2} T^{2} \zeta^{2}\right), B^{\prime}:=\sqrt{C_{f}} K(1+T) \varepsilon^{2}$. The terms caused by $\nabla^{2}(\varphi+t \psi)$ can be estimated by $3 K(1+T) \varepsilon^{3}$, which is much smaller than $K(1+T) \varepsilon^{2}$ if $\varepsilon$ is sufficiently small. These cubic terms have been neglected in the above estimate.

To sum up, we have $\|\mathcal{L} w\| \leq A\|w\|+B+\left(A^{\prime}\|w\|+B^{\prime}\right)^{2}$.

We fix $(\zeta, T)$ and introduce a number $r$ as in the following ( $\zeta$ satisfying the condition indicated above), where $\mu>0$ is a small parameter:

$$
\zeta=2 e^{2} \varepsilon, \quad T=\frac{\mu}{\varepsilon}, \quad r=\frac{2 B}{1-2 A} .
$$

We have $0<A<1 / 3$ and $r>6 B>0$ if $\mu$ is sufficiently small. If $0<\varepsilon<1$, there exist positive constants $C_{A}, C_{B}, C_{r}, C_{A^{\prime}}$ and $C_{B^{\prime}}$ such that

$$
\begin{aligned}
& A=C_{A} \mu^{2}, \quad B=C_{B}\left(\varepsilon^{3}+\mu \varepsilon^{2}\right), \\
& A r+B=r / 2, \quad 0<r \leq C_{r}\left(\varepsilon^{3}+\mu \varepsilon^{2}\right), \\
& A^{\prime} \leq C_{A^{\prime}} \mu^{2} \varepsilon^{-1}, \quad B^{\prime}=C_{B^{\prime}}\left(\varepsilon^{2}+\mu \varepsilon\right) .
\end{aligned}
$$

Note that $T^{2} \zeta^{2}$ is much smaller than $T^{2} \zeta$. It means that the terms related to $\nabla^{2}$ are much smaller than those related to $\nabla$.

There exists a positive constant $C_{1}$ such that

$$
\left(A^{\prime} r+B^{\prime}\right)^{2} \leq C_{1} \varepsilon^{2}(\varepsilon+\mu)^{2} .
$$


On the other hand, $r$ can be estimated from below, and there exists a positive constant $C_{2}$ such that

$$
C_{2} \varepsilon^{2}(\varepsilon+\mu) \leq r .
$$

Therefore if $\varepsilon+\mu$ is sufficiently small, we have

$$
A r+B+\left(A^{\prime} r+B^{\prime}\right)^{2}=\frac{r}{2}+\left(A^{\prime} r+B^{\prime}\right)^{2} \leq r .
$$

When $\zeta, T$ and $r$ are as in $(*)$, let $B(r ; T, \zeta) \subset \mathcal{G}_{T, \zeta}(\Omega)$ be the closed ball of radius $r$ centered at 0 . The above calculation shows that $\mathcal{L}$ is a mapping from $B(r ; T, \zeta)$ to itself if $\varepsilon+\mu$ is sufficiently small.

Next we shall show that $\mathcal{L}$ is a contraction mapping. Take $w_{1}, w_{2} \in B(r ; T, \zeta)$ with $r, T, \zeta$ as in (*). We have

$$
\mathcal{L}\left(w_{1}\right)-\mathcal{L}\left(w_{2}\right)=P \partial_{t}^{-2}\left(w_{1}-w_{2}\right)+f\left(\overrightarrow{\tau_{1}}\right)-f\left(\overrightarrow{\tau_{2}}\right),
$$

where $\overrightarrow{\tau_{j}}=\nabla^{1,2}\left(\partial_{t}^{-2} w_{j}+\varphi+t \psi\right)$.

Then by Propositions 2.4 and 2.5, we have

$$
\begin{aligned}
& \left\|\mathcal{L}\left(w_{1}\right)-\mathcal{L}\left(w_{2}\right)\right\| \\
& \leq A\left\|w_{1}-w_{2}\right\|+C_{f}^{\prime}\left\|\overrightarrow{\tau_{1}}-\overrightarrow{\tau_{2}}\right\|_{N}\left(\left\|\overrightarrow{\tau_{1}}\right\|_{N}+\left\|\overrightarrow{\tau_{2}}\right\|_{N}\right) .
\end{aligned}
$$

Since $T^{2} \zeta^{2}$ is much smaller than $T^{2} \zeta$ and $T^{2} \zeta \leq C_{2} \mu^{2} \varepsilon^{-1}$ for some $C_{2}$, we have

$$
\begin{aligned}
\left\|\overrightarrow{\tau_{1}}-\overrightarrow{\tau_{2}}\right\|_{N} & \leq \max \left(C_{-2,1} T^{2} \zeta, C_{-2,2} T^{2} \zeta^{2}\right)\left\|w_{1}-w_{2}\right\| \\
& =C_{2} \mu^{2} \varepsilon^{-1}\left\|w_{1}-w_{2}\right\| .
\end{aligned}
$$

On the other hand, since $\left\|w_{j}\right\| \leq r \leq C_{r}\left(\varepsilon^{3}+\mu \varepsilon^{2}\right)$, there exists $C_{3}>0$ such that

$$
\left\|\overrightarrow{\tau_{j}}\right\| \leq C_{-2,1} T^{2} \zeta\left\|w_{j}\right\|+K(1+T) \varepsilon^{2} \leq C_{3}\left(\varepsilon^{2}+\mu \varepsilon\right) .
$$

Hence for some $C_{4}>0$,

$$
\frac{\left\|\mathcal{L}\left(w_{1}\right)-\mathcal{L}\left(w_{2}\right)\right\|}{\left\|w_{1}-w_{2}\right\|} \leq C_{A} \mu^{2}+2 C_{4} \mu^{2}(\varepsilon+\mu) .
$$

We find that $\mathcal{L}$ is a contraction mapping if $\mu+\varepsilon$ is sufficiently small. Its fixed point

$$
w \in \mathcal{G}_{T, \zeta}(\Omega) \subset \mathcal{C}(T ; A(\Omega))
$$

gives us a solution $u(t, x)=\partial_{t}^{-2} w(t, x)+\varphi(x)+t \psi(x) \in \mathcal{C}^{2}(T ; A(\Omega))$.

Proof of Theorem 1.2, Local uniqueness follows from the Cauchy-Kovalevskaya theorem, and we can extend it by analytic continuation.

Now we sketch the proof of existence. A complex-analytic function on $B_{T} \times U$ is said to be an element of $\mathcal{G}_{T, \zeta}^{\mathbb{C}}(U)$ if there exists a constant $C>0$ such that

$$
\forall \alpha \in \mathbb{N}^{n}, \forall t \in B_{T}, \quad \sup _{x \in U}\left|\partial^{\alpha} u(t, x)\right| \leq C \zeta^{|\alpha|} D^{|\alpha|} \varphi(|t| / T) .
$$

It can be proved that $\mathcal{G}_{T, \zeta}^{\mathbb{C}}(U)$ is a Banach algebra. The theorem can be proved in the same way as in the real case. 


\section{REFERENCES}

[1] D'Ancona P. and Spagnolo S., Global solvability for the degenerate Kirchhoff equation with real analytic data, Invent. Math., 108(1992), 247-262. MR.1161092(93h:35131)

[2] Georgiev V., "Semilinear hyperbolic equations", Mathematical Society of Japan, Tokyo, 2000. MR:1807081 (2001k:35003)

[3] Daniel G. and Mustapha M., Problème de Cauchy pour des equations de Kirchhoff generalisées, Comm. Partial Differential Equations, 23(1998), 761-776. MR1632815 (99c:35160)

[4] Hörmander L., "Lectures on nonlinear hyperbolic differential equations", Springer-Verlag, Berlin, Heidelberg, 1997. MR1466700 (98e:35103)

[5] Kichenassamy S., "Nonlinear wave equations", Marcel Dekker, New York, 1996. MR1362547 (96j:35001)

[6] Wagschal C., Le Problème de Goursat non linéaire, J. Math. Pures Appl., 58(1979), 309-337. MR0544256 (82m:35024)

[7] Wakabayashi S., The Lax-Mizohata theorem for nonlinear Cauchy problems, Comm. Partial Differential Equations, 26(2001), 1367-1384. MR1855282 (2002j:35073)

Department of Physics, Kwansei Gakuin University, Gakuen 2-1, Sanda, Hyougo 6691337, JAPAN

E-mail address: yamane@ksc.kwansei.ac.jp 